\title{
The Dissolution of Bar-Hillel-Carnap Paradox BY SEMANTIC INFORMATION THEORY BASED ON A PARACONSISTENT LOGIC
}

\author{
SAMIR GORSKY \\ Department of Philosophy, Federal University of Rio Grande do Norte, BRAZIL \\ samirgorsky.ufrn@gmail.com
}

\begin{abstract}
Several logical puzzles, riddles and problems are defined based on the notion of games in informative contexts. Hintikka argues that epistemology or the theory of knowledge must be considered from the notion of information. So, knowledge cannot just be based on the notions of belief and justification. The present proposal will focus on the logical structure of information, and not only on the quantification of information as suggested by Claude A. Shannon (1916-2001) (Shannon 1948). In many cases, the information bits, although seemingly or factually contradictory, are quite relevant. The paraconsistent systems of logic offer a formalization of reasoning that can support certain contradictions. The well-known "Bar-Hillel-Carnap Paradox" (Bar-Hillel, 1964) causes embarrassment when it concludes that the informational content of a contradiction would be maximum, opposing the traditional notion that the semantic information must be true, and that contradictions are necessarily false.
\end{abstract}

Keywords: Paraconsistent logics $\bullet$ coding and information theory $\bullet$ problems with incomplete information

\section{The theory of semantic information}

The quantification of information proposed by Shannon is supported by terms of probability. In similar terms, the semantic approach of information theory requires a purely logical notion of probability. The semantic information is given by an inverse relationship between probability and information (see formula 8 below). This approach was initially defended by Carnap and Bar-Hillel (1964) (first published in 1953). Later, Kemeny (1953), Smokler (1966), Hintikka and Suppes (Hintikka, 1970) contributed to the development of the initial proposal. Dretske (1981) complemented the basis of the semantic information theory. ${ }^{1}$

Some researchers consider the measure of information an important branch of research of information theory. Another relevant topic of this theory is the relationship between information and knowledge. From this point of view, we can consider a 
way to address epistemological questions in the light of information theory. So, here is an epistemology based on the concept of information.

In everyday speech we say we have received information, when we know something that we did not know before: when "what we know" has changed. If then we were able to measure "what we know", we could talk meaningfully about the amount of information we have received, in terms of the measurable change it has caused. This would be invaluable in assessing and comparing the efficiency of methods of gaining or communicating information. Information Theory is concerned with this problem of measuring. (Mackay 1950, p.9)

With the development of research in information theory in the mid-twentieth century, it became possible to quantify information from probability calculus (cf. Shannon and Weaver, 1974, Mathematical Theory of Communication). Jaakko Hintikka, inspired by the work of Yehoshua Bar-Hillel and Rudolf Carnap, proposed an extension of the theory of semantic information related to the underlying logic of informational scenarios and its descriptions (Hintikka, 1970).

According to Floridi (2013) certain philosophical theories about semantic information aim to connect this concept to other forms of complex phenomena, such as epistemic, mental, doxastic, etc. A theory of semantic information from the point of view of its factual content or structure was worked by Dretske (1981) and by Barwise and Seligman (1997). This approach was also known as the naturalization of information.

Formally, both the statistical information theory and the semantic information theory have elements in common. These theories are defined, or may be defined, in terms of an appropriate concept of probability.

The connection made between information and probability is the same for the two cases:

$$
\inf (h)=\log _{2} p(h)
$$

where $\inf (h)$ is the measure of information about an event $h$, and $p$ are the probability measure in question. From equation (1), one can obtain the known expression of entropy:

$$
-\sum_{i} p_{i} \log p_{i}
$$

This formula is applied in cases where there is a number of exclusive alternatives with probability $p_{i}$, such that $i=1,2,3, \ldots$.. Carnap, in (1993), Logical Foundation of Probability, pp.29-36, introduces two concepts of probability: probability ${ }_{1}$ and 
probability $_{2}$. This distinction between such concepts refers to a possible contrast between statistical information theory and semantic information theory. This difference can be explained through the following terms: in agreement with statistical information theory, probability should be interpreted in terms of frequencies of occurrence of events; on the other hand, according to the semantic information theory, we assume a logic formulation of probability.

\section{The Bar-Hillel-Carnap Paradox and the Scandal of Deduction}

It is usual to qualify a deductive inference as being analytical. In informational terms this characteristic can be formulated as follows: the information contained in the conclusion is a subset of the information contained in the premises, i.e., the information contained in the conclusion is already contained in the premises. In terms of truth functions, i.e., functions from a set of truth values to truth values, it is common to repeat the claim that the conclusion of a valid deduction keeps the true value contained in the premises, or that the set of possible worlds that verifies the set (or the conjunction) of all premises is a subset of the set of possible worlds that verifies the conclusion. The reconciliation between the two views can be done via the semantic perspective of information theory.

The Inverse Relationship Principle states that the concepts of information and unpredictability are similar. More precisely, there is an inverse relationship between the probability that a proposition $P$ is shown to be true, and the sum of information (semantic information) supported by $P$.

According to D. Agostino and Floridi (2009), any analysis of semantic information that supports the Inverse Relationship Principle touches on two major difficulties: the Bar-Hillel-Carnap Paradox and the Scandal of Deduction.

The Inverse Relationship Principle maintains that the less probable or possible a proposition is, the greater the amount of semantic information carried by it. It follows that the contradiction is a kind of message that contains the greatest amount of semantic information. Many researchers consider this conclusion as being unpalatable (D. Agostino and Floridi, 2009). Bar-Hillel and Carnap are among the first researchers to make explicit this result that in the texts of Floridi is called the Bar-Hillel-Carnap Paradox (cf. Bar-Hillel, 1964).

According to Floridi and D'Agostino (2009), despite being an unfortunate result, the paradox is an inevitable logical consequence for any quantitative theory of weak semantic information (called "weak" because the truth values do not have influence in obtaining this result). There are some attempts to overcome this paradox, consisting of trying to show that Semantic Information Theory is based on a weak semantic 
principle (cf. D. Agostino and Floridi, 2009). So it would be natural to extend this proposal to the logics that are able to explain some semantic aspects within their own language, for example, the paraconsistent logics.

Now, if we look at tautologies and consider their probabilistic values, we see that their information content is zero. As deductions always need a finite number of premises, each deduction can also be seen as a tautological conditional statement where the conjunction of all the premises is the antecedent and the conclusion the consequent. Once all tautologies have probability 1 (that is, are true in all possible worlds) and have null informative content (by the Inverse Relationship Principle), then all deductions have zero information content.

The following question, based on the above argument, leads us to what Hintikka has called the Scandal of Deduction: in which sense can we say that the valid logical deductions provide us with new information? Or, in other words, how could we ever be surprised in front of analytical deductions?

The present paper deals only with the case of the Bar-Hillel-Carnap Paradox. The treatment of the Scandal of Deduction will be indicated for future work.

\section{The generalization of semantic information theory}

The most acceptable measure of information deals with selective information. Other measures are possible, but this measure will suffice for the purposes of this paper. (For further information measures, see Mackay 1950, The Nomenclature of Information Theory). One of the questions that allow us to understand the definition of selective information is this: what degree of difficulty exists in identifying a particular element of a given set? It is thus natural, for the context for applications, to measure the unit of information in terms of elements and sets.

In a set $C=\left\{n_{1}, n_{2}, n_{3}, \ldots, n_{k}\right\}$ with $k$ elements, the information that determines a particular element $n_{i}$ will have $\log _{2} k$ bits. This is because any element in this set can be unequivocally encoded by a sequence of symbols ranging in $D$, where $D$ is a set with two different digits. Each position of codification should be filled out with only one digit, $d_{i} \in D$. In order to be effective, we will only consider finite sequences of symbols (or, in other words, finite sets). Another way of explaining the measure is through a game consisting of two agents, $A$ and $B$, and playing with the set $C=$ $\left\{n_{1}, n_{2}, n_{3}, \ldots, n_{k}\right\}$. One of the players (let's say $A$ ) must choose one of the elements of $C$, while the other player $(B)$ will have to find out what was the chosen element. For this, $B$ should ask questions like "Is the chosen element $y$ ?", "Is the chosen element between $x$ and $z$ ?", "Is the chosen element greater than or equal than $w$ ?" and so on, where $x, y, z$ and $w$ range over the set $C$. The player $A$ will have to answer "yes" or "no" according to the case. The best strategy for $A$ is the known binary search 
algorithm. If we determine that the average number of questions needed to find out the chosen number is a measure of information for this scenario, then the measure of the difficulty to find the number chosen or drawn can also be determined. The higher this measure is, the higher the initial uncertainty over the chosen element. Likewise, we can quantify the "amount" of surprise involved here. The greater the measure of uncertainty of an element in a set is and the smaller the number of questions that were actually used is, the more surprise the discovery of the number generates (cf. Hintikka 1970, On semantic information). This happens because the measure of surprise depends on the unexpected hit. That is why it is considered more surprising to hit a number in a million than one in a thousand (both with 3 questions).

"When we receive information, it causes a change in the symbolic picture or representation which we would use to depict what we know. It is found the changes in representations can be measured; so "amount of information", actually in more than one sense, can be given numerical meaning. It is as if we had discovered how to talk quantitatively about size, through discovering its effects on measuring-apparatus." (Mackay 1950, p 9).

In Watanabe (1969), Knowing and Guessing: A formal and Quantitative Study, we find the following definition:

$$
I=D I=\operatorname{ign}(\varepsilon)-\operatorname{ign}^{\prime}(\varepsilon)=-\sum_{i} p_{i} \log p_{i}
$$

where $I$ is the quantity of information, which coincides with the amount of decrease in ignorance $D I$, and where $\operatorname{ign}(\varepsilon)$ and $\operatorname{ign}^{\prime}(\varepsilon)$ are, respectively, the quantity of ignorance of a proposition before and after the fact of the proposition's occurrence. This formula allows us to interpret epistemology from the point of view of information. Scientific discovery is guided by a theoretical construct that allows one to simulate the unknown within the known. The constituent information (previously discovered) of the scenario is reconfigured within the previous structure of information. The quantity of surprise can be understood as the amount of information liberated by such an event. The mind (or the imagination) has the ability to simulate the behaviors of these changes and therefore conceive the characteristics of theories or of complex scenarios consistent with other theories or scenarios. Thus, despite the possibility confirmed above, there is not still a general theory that manages the structure of information ignored or quasi-known (or conceivable).

As we have seen, Watanabe (1969), Knowing and Guessing: A formal and Quantitative Study, shows a relationship between the measurement of information and the quantity of ignorance about an event before its actualization or realization. Depending on the strategy taken, the amount of information contained in the assertion "the chosen element is $n_{j}$ " may differ (cf. Edwards, 1964). 
In view of the fact that the concept of information is being treated based upon the number of questions or answers needed to identify unequivocally one element of a certain set, we can say that this is an approach related with the viewpoint of erotetic logic, or the "logic of questions".

In 1976, the mathematician Stanislaw Ulam proposed a riddle involving a structure of search as described above. However, among the possible answers, the player who was answering the questions could lie one, two or more times. The problem defined by Ulam is to decide how many questions are needed to identify the number drawn under this new condition. In (Mundici 1990, Two Papers on Ulam's Logic with Lies), Mundici shows that an adequate logic to the Ulam's game is the Łukasiewicz's Logic. Similar games can be designed where the base of reasoning is incorporated with elements from non-classical logics. Some studies may be carried on a generalization of information theory from paraconsistency and the relationship with elements of the study of probabilities. For example, Walter A. Carnielli in (2009), Uma lógica da modalidade econômica?, proposes a reformulation of Bayes theorem taking the paraconsistent logic Cie as the underlying logic.

This paper concentrates on the possibility of applying information theory for analyzing scenarios that were previously outside of its scope. For this, we take the semantic point of view of information theory. Based on classical logic, we try to extract a logical structure of certain information contexts. One of the primary goals to be reached when considering the logical basis of an informative context is to identify the possibility of distinguishing the different alternatives by means of the expressive sources available, or more simply, through the language in use. The more alternatives a sentence admits, the more probable it will be (in some "purely logical" sense). Thus, it is reasonable to say that the sentence $(P \vee Q)$ carries more information than the sentence $(P \wedge Q)$, even in view of the additional information that both are true. In this case, there are more possible worlds or interpretations that model $(P \vee Q)$ than worlds or interpretations that model $(P \wedge Q)$.

Based on the idea that puzzles are connected to certain types of games in defined informative scenarios (or descriptions of states or possible worlds), we define logicalformal descriptions, that we call scenarios (following Jakko Hintikka, 1970).

In logical terms (i.e., in terms of the classical propositional logic), the different possible cases (or descriptions of the world) are defined from the idea of constituents and these have the following form:

$$
( \pm) P_{1} \wedge( \pm) P_{2} \wedge( \pm) P_{3} \wedge \ldots \wedge( \pm) P_{k}
$$

Each symbol $( \pm)$ may be substituted by $\neg$ or deleted, thus generating several possibilities of constituents from the given set of sentences. All constituents have size $k$. The difference between the constituents is determined by the different ways in 
which the negation is distributed between sentences. Therefore, the total number of constituents will be $2^{k}$.

Each description that admits one of the alternatives described by constituents excludes the others. It will be true if any of the alternatives allowed are true and false otherwise. Each description can be represented as a disjunction of some (possibly all) constituents, provided that at least one of these is admitted. As a formal example, let us take the following description:

$$
h=C_{1} \vee C_{2} \vee C_{3} \vee \ldots \vee C_{w(h)}
$$

In this case, $h$ is the description, and its scope is $w(h)$. When $h$ is inconsistent, $w(h)=0$. In light of these definitions, it is very simple to identify the logical concept of probability linked to appropriate measures of information. Obviously, the constituents are the representatives of different atomic symmetrical events that are taken as the basis for this measure of information. A description $h$ is more probable or has more chances to match the facts as more alternatives represented by constituents it admits or, in other words, the higher $w(h)$ is. Thus, it is presumed that the measure of probability in the logical sense can be defined as follows:

$$
p(h)=\frac{w(h)}{2^{k}}
$$

Obviously, this is a probability of a finite set. From (1) and (6) it is possible to obtain the following measure of information:

$$
\inf (h)=-\log p(h)=-\log \left(\frac{w(h)}{2^{k}}\right)=k-\log w(h)
$$

In this formula, it is assumed that the base of the logarithm is 2 . It is easy to see that $-\log p(h)=-\log \left(\frac{w(h)}{2^{k}}\right)$, once $p(h)=\frac{w(h)}{2^{k}}$ by 77 . Hence, by definition one arrives at $k-\log w(h){ }^{2}$ Such an equation also provides a very intuitive idea about the concept of information: the more accurate or timely a sentence, the more informative it is. Thus, the more alternatives a statement excludes, the more informative it is (This idea has been emphasized in particular by Karl Popper).

In the case of an inconsistent description, $w(h)=0$. Since the logarithm of 0 is not defined, the value of $\inf (h)$ will be considered from the limit of the function, namely $\lim _{x \rightarrow 0}\left(\log _{2} x\right)=-\infty$. This interpretation is reasonable, since the inconsistency is related to the maximum limit of "understandable" information contained in a description.

Therefore, a further and interesting way to look at the measure of information is through excluded alternatives by the description. This notion is defined as the content 
of a description or $\operatorname{cont}(h)$ :

$$
\operatorname{cont}(h)=\frac{2^{k}-w(h)}{2^{k}}=\frac{2^{k}}{2^{k}}-\frac{w(h)}{2^{k}}=1-p(h)
$$

The result $1-p(h)$ is obtained by definition of $p(h)$ in 6 , and cont $(h)$ can be seen as a measure of information that a given number of alternatives excludes. We can also think of $\operatorname{cont}(h)$ as being the probability of the excluded events. $2^{k}$ is the total number of different constituent, so $2^{k}-w(h)$ is the number of excluded alternatives.

Let $1-p(w(h))$ be the probability of excluded alternatives:

$$
1-p(w(h))=\frac{2^{k}-w(h)}{2^{k}}
$$

It is noticed immediately that there is a relationship between this definition and the primary definition of information. In fact, this is a perfectly reasonable measure of information conveyed by $h$. Conversely, one can define inf $(h)$ based on $\operatorname{cont}(h)$ as follows:

$$
\inf (h)=\log \left(\frac{1}{1-\operatorname{cont}(h)}\right)
$$

The previous relationship between the two measures is quite interesting. It is often argued that the point of view of the logical structure of the information herein sometimes is based on the first definition, sometimes on another one. It is thus suggested that cont $(h)$ can be regarded as the substantial information measurement conducted by a description; $\inf (h)$ is regarded as a measure of surprise, that is, it measures how unexpected its truth is (cf. Hintikka, 1970).

Some insights about the difference between the functions cont and inf may be given by the results below:

$$
\operatorname{cont}(h \wedge g)=\operatorname{cont}(h)+\operatorname{cont}(g)
$$

if and only if $(h \vee g)$ is logically true;

$$
\inf (h \wedge g)=\inf (h)+\inf (g)
$$

if and only if $h$ and $g$ are independent with respect to the measure of probability $p$ defined by 6 ;

$$
\inf (h)=\operatorname{cont}(h)=0
$$

if and only if $h$ is logically true. 
The above equations provide the framework for a logical theory of (semantic) information based on classical propositional logic, because they define concepts such as probability, surprise and information from scenarios built only with atomic sentences and logical connectives (conjunction, disjunction and negation). This was presented in Hintikka (1970) based on the work of Rudolf Carnap where there are extensions of this case to monadic first order logic.

In this context, both the Scandal of Deduction and the Bar-Hillel-Carnap Paradox holds. The measure of semantic information of an inconsistent description tends to infinity and of the tautological descriptions are 0 .

Taking into account that these scenarios can be used in the formal description of puzzles or riddles, we generalize the above cases based on some non-classical logics. At first, the generalization will be proposed for the case of paraconsistent logic LFI1.

\section{Information theory approach under a paraconsistent view}

In the context of formal symbolic languages, it is said that a deductive theory is consistent if it has no contradictory theorems; otherwise the theory is called inconsistent.

Following the notation and constructions contained in Carnielli, Coniglio and Marcos (2007), Logics of Formal Inconsistency, a theory $\Gamma$ is contradictory if satisfies:

$$
\exists \alpha(\Gamma \Vdash \alpha \text { and } \Gamma \Vdash \neg \alpha)
$$

It is know that there are important kinds of reasoning that makes use of contradictory information in the same scenario, but without trivializing the underlying deductive system. Theories that support some contradiction without trivializing the system are called "non-explosives". Several puzzles can be characterized from this property, i.e., their solutions are achieved based on the use of reasoning about a set involving contradictory information, albeit the correct solution is not trivial and in many cases should be considered objectively. In science, philosophy, physics and even mathematics, there are some theories that contradict others or which are partly contradictory. In many of these cases the contradiction is shown as a relevant information source since it does not trivialize the systems. Therefore, the main objective in studying such cases is not to remove contradictions or conflicting theories, but to adapt ourselves to these reasoning scenarios in order to obtain more effective research methods.

Several researchers in logic have developed important works on paraconsistency. Among these works we can cite Logics of Formal Inconsistency, published in volume 14 of the Handbook of Philosophical Logic, edited by Dov Gabbay and Franz Guenthner (Carnielli, Coniglio and Marcos, 2007). The Logics of Formal Inconsistency are systems in which the C-Systems can be interpreted as a subclass (henceforth LFIs), namely: they are systems in which consistency can be expressed by a unary operator. 
Classical theories are characterized as explosives in the broadest sense of the term. The LFIs, by internalizing the notions of consistency and inconsistency at the level of object language, allow graduations of non-explosives contradictions in the theories of their systems. With this, it becomes possible to treat paraconsistent reasoning in various degrees. Then, the paraconsistency can be defined as the study of contradictory and non-trivial theories.

Clearly, the concept of paraconsistency is related to the properties of negation within a given logic. The works of Arnon Avron (2002) On negation, completeness and consistency, Jean-Yves Béziau (1994) Theórie legislative de la negation pure and Wolfrang Lenzen (1998), Necessary conditions for negation-operators (with particular applications to paraconsistent negation) deal with the role negation in paraconsistent logics and João Marcos (2004), Nearly every normal modal logic is paranormal presents a summary of the ideas contained in the preceding articles.

A first approximation between paraconsistency and informative scenarios as described above is made with the logic LFI1, also known as $\mathbf{J}_{3}$ or CLuNs (cf. Carnielli, Marcos, and Amo 2000, Formal inconsistency and evolutionary databases).

In the present case, inconsistency and contradiction may be taken as identical. The symbol $\bullet$ characterizes a contraditory information $A$ as follows: if $A$ is a contraditory information, then holds $\bullet$. A contradictory information may also be presented as $A \wedge \neg A$. Therefore, it holds for the equivalence $\bullet A \leftrightarrow A \wedge \neg A$.

Informational contradictory scenarios are informative scenarios that for some sentence $P \in$ Cenc, where Cenc is the set of constituents of the scenario, $\bullet P$ holds. This generalization of information theory is the result of information theory about informational contradictory scenarios (or ICS). If the logic underlying these scenarios has the paraconsistent characteristic of being non-explosive, then such scenarios will be called suitable informational contradictory scenarios (or SICS).

We present below the formal definition of a paraconsistent constituent. Accordingly, descriptions of the world (in terms of possible worlds) are analyzed in a generalized theory of SICS, taking into account contradictory constituents:

$$
(\dot{ \pm}) P_{1} \wedge(\dot{ \pm}) P_{2} \wedge(\dot{ \pm}) P_{3} \wedge \ldots \wedge(\dot{ \pm}) P_{k}
$$

The symbols $\dot{ \pm}$ must be replaced by a single symbol contained in the following set, $S=\{\bullet, \neg\}$, or completely erased according to the case to be described. The symbol $\bullet P$, in the reading of the logics of formal inconsistency, means that $P$ is inconsistent. In particular we will be dealing with the three-valued paraconsistent logic LFI1 (see Carnielli, Marcos, and Amo, 2000). In this case $\bullet P$ holds iff $P$ (and $\neg P$ ) is partially true. The case $\bullet P_{i}, i \in\{1,2, \ldots, k\}$, then, admits simultaneously the cases $P_{i}$ and $\neg P_{i}$.

There are thus three possibilities for each $P \in$ Cenc, namely: $P$ holds, $\bullet P$ holds 
or $\neg P$ holds. The total number of constituents is $3^{k}$. The descriptions, unlike the classical case, admit intersections of cases, i.e., some descriptions may overlap. The descriptions, however, are partially exclusive. This happens because the paraconsistency occurs in cases that there is conflicting information without trivialization. The constituents with contradictory information may be partially the same as information present in constituents that have no contradictions. Likewise, each description may be represented as a disjunction of some (possibly all) the constituents, provided that they admit at least one of these:

$$
h^{\text {Par }}=C_{1}^{\text {Par }} \vee C_{2}^{\text {Par }}, \ldots, C_{w\left(h^{\text {Par }}\right)}^{\text {Par }}
$$

where $h^{\text {Par }}$ is the sentence that describes the scenarios (possibly paraconsistent) and $w\left(h^{\text {Par }}\right)$ its range. Now, if $h^{\text {Par }}$ is contradictory (all constituents have contradictory descriptions $\left.\bullet P_{i}\right)$, then we do not necessarily have the equation $w\left(h^{\text {Par }}\right)=0$. The idea in this fact is that a contradictory description is more informative than the absence of description. It is possible in this context to extend the definitions of the key concepts of the theory of logical or semantic information to non-classical (paraconsistent) cases. The logical concept of probability can thus be set up to paraconsistent contexts as follows:

$$
p\left(h^{\text {Par }}\right)=\frac{w\left(h^{\text {Par }}\right)}{3^{k}}
$$

When it is established that $\bullet P_{i}, i \in 1,2, \ldots$ appears in a description, it is concluded that the information contained in the proposition cannot be sustained in a non-contradictory way. Thus, the description requires a local revision if the contradictory information is relevant, otherwise only the other items of description will be taken into account.

From the classic definition of information one obtains the appropriate measurement of information based on the paraconsistent logic probability (LFI1) with the following expression:

$$
\inf \left(h^{\text {Par }}\right)=-\log _{2} p\left(h^{\text {Par }}\right)=-\log _{2}\left(\frac{w\left(h^{\text {Par }}\right)}{3^{k}}\right)=k \log _{2} 3-\log _{2} w\left(h^{\text {Par }}\right)
$$

The measure $\operatorname{cont}^{\text {Par }}\left(h^{\text {Par }}\right)$ is identical to the propositional classic case if in the description there does not appear any sentences of the form $\bullet P_{i}$. In general, has the following form:

$$
\operatorname{cont}^{\text {Par }}\left(h^{\text {Par }}\right)=\frac{3^{k}-w\left(h^{\text {Par }}\right)}{3^{k}}=\frac{3^{k}}{3^{k}}-\frac{w\left(h^{\text {Par }}\right)}{3^{k}}=1-p\left(h^{\text {Par }}\right)
$$

Given the above definitions of the elements of information theory to the case of an underlying paraconsistent logic, it is possible to define the relationship between 
inconsistent sentences and a description of the sets $K_{j}$ of the elements known by $j$. When $\bullet P_{i}$ is found, one can conclude that $P_{i} \in K_{j}$ and $\neg P_{i} \in K_{j}$, where $K_{j}$ is the set of propositions known by $j$. However, it is reasonable to conclude that when $P_{i}$ and $\neg P_{i}$ is found, the conclusion is $\bullet P_{i} \in K_{j}$.

Semantic information is measured by taking up a logical scenario model of knowledge. Thus, the amount of information is a measure of the "impact" of the sentences on the known scenario. However, the intermediate stages (in which the information received is being added to the scenario) could sustain possible contradictions. From this one cannot derive a trivialization of the set of sentences known by an agent. Therefore, the treatment of these intermediate states is conveniently done through paraconsistent logical systems. As was shown above, this treatment provides a new measure of information for scenarios with contradictory sentences. We also show that this new way to measure information (from contradictory scenarios in the system LFI1) is an alternative to the Bar-Hillel-Carnap Paradox. According to the results, if the logic of the scenario is replaced by paraconsistent logic, then the amount of information in a contradictory scenario is not infinite, and therefore the theoretical framework of the theory of semantic information guarantees that such a measure is computable. In this way, the so-called Bar-Hillel-Carnap Paradox vanishes.

The case of the Scandal of Deduction was not considered here. However, since the substitution of the basic logic was fundamental for the dissolution of the case of the paradox, it is convenient to affirm that the treatment of the case of the Scandal of Deduction could be done using a basic intuitionist logic. In this case, one has the possibility of treating tautological cases in a more constructive way. So, the case of the Scandal of Deduction will be considered in future works.

\section{References}

Avron, A. 2002. On negation, completeness and consistency. In: D. Gabbay; F. Guenthner (eds.) Handbook of Philosophical Logic, pp.287-319. 2nd ed. volume 9. Kluwer Academic Publishers.

Béziau, J.-Y. 1994. The théorie legislative de la negation pure. Logique et Analyse 147-148: 209-225.

Bar-Hillel, Y. 1964. Language and Information. Jerusalem: Addison-Wesley and The Jerusalem Academic Press.

Barwise, J.; Seligman, J. 1997. Information Flow: The Logic of Distributed Systems. Cambridge Tracts in Theoretical Computer Science. Cambridge University Press.

Carnielli, W. A. 2009. Uma lógica da modalidade econômica? Revista Brasileira de Filosofia 232: 209-225.

Carnielli, W. A.; Coniglio, M.; Marcos, J. 2007. Logics of formal inconsistency. In: Gabbay, D.; F. Guenthner (eds.) Handbook of Philosophical Logic, pp.15-107. 2nd. edition, volume 14. Springer-Verlag. 
Carnielli, W. A.; Marcos, J.; Amo, S. 2000. Formal inconsistency and evolutionary databases. Logic and Logical Philosophy.

Agostino, M. D.; Floridi, L. 2009. The enduring scandal of deduction. Synthese 167(2): 271315.

Dretske, F. I. 1981. Knowledge \& the Flow of Information. Bradford Books. Mit Press.

Edwards, E. 1964. Introdução à Teoria da Informação. São Paulo: Cultrix.

Floridi, L. 2013. Semantic conceptions of information. Stanford Encyclopedia of philosophy.

Hintikka, J. 1970. On semantic information. In: J. Hintikka; P. Suppes (eds.) Information and Inference. Dordrecht, Holland: D. Reidel Publishing Company.

Kemeny, J. G. 1953. A logical measure function. Journal of Symbolic Logic 18: 284-308.

Lenzen, W. 1998. Necessary conditions for negation-operators (with particular applications to paraconsistent negation). In: A. Besnard; P. Hunter (eds.) Reasoning with Actual and Potential Contradictions, pp.211-239. Dordrecht: Kluwer.

Mackay, D. M. 1950. The nomenclature of information theory.

Marcos, J. 2004. Nearly every normal modal logic is paranormal. Technical report 1049001, CLC, Department of Mathematics, Instituto Superior Técnico, Lisbon, PT. Submitted for publication. Preprint available at URL $=$ http://wslc.math.ist.utl.pt/ftp/ pub/MarcosJ/04-M-Paranormal.pdf

Mundici, D. 1990. Two papers on Ulam's logic with lies. Aila Preprint 3.

Shannon, C. A. 1948. Mathematical theory of communication. Reimpresso com correções pelo The Bell System Technical Journal 27: 379-423, 623-656.

Smokler, H. 1966. Informational content: A problem of definition. The Journal of Philosophy 63(8): 201-211.

Shannon, C. E.; Weaver, W. 1974. The mathematical theory of communication. Urbana, Illinois: The University of Illinois Press.

Watanabe, S. 1969. Knowing and Guessing: A formal and Quantitative Study. New York: John Wiley \& Sons, INC.

\section{Notes}

1 The present paper was written with important participation and assistance of Professor Walter Carnielli, who accompanied the development of the research presented here and contributed with technical review and comments.

2 The logarithm of a fraction is the difference of the logarithm of its terms. 